\title{
Susceptibility-Weighted Magnetic Resonance Imaging for the Detection of Cerebral Microhemorrhage in Patients With Traumatic Brain Injury
}

\author{
Yukinori AKIYAMA*,**, Kei MIYATA**, Kuniaki HARAdA*, \\ Yoshihiro MINAMIDA*, Tadashi NONAKA*, Izumi KOYANAGI*, \\ Yasufumi ASAI**, and Kiyohiro HOUKIN* \\ Departments of ${ }^{*}$ Neurosurgery and ${ }^{* *}$ Critical Care Medicine, \\ Sapporo Medical University School of Medicine, Sapporo, Hokkaido
}

\begin{abstract}
The sensitivity of susceptibility-weighted magnetic resonance (MR) imaging was compared with conventional MR sequences, including $\mathrm{T}_{2}{ }^{*}$-weighted imaging, and computed tomography for the detection of cerebral hemorrhages in 15 patients with head injury. Susceptibility-weighted imaging detected a mean of $76 \pm 52$ (total 1132) hypointense spotty lesions, compared to a mean of $21 \pm 19$ (total 316) detected by $\mathrm{T}_{2}{ }^{*}$-weighted imaging $(\mathrm{p}<0.0001$, paired $\mathrm{t}$-test). Susceptibility-weighted imaging is extremely sensitive for the visualization and detection of microhemorrhages.
\end{abstract}

Key words: susceptibility-weighted magnetic resonance imaging, cerebral microhemorrhage, brain injury

\section{Introduction}

The standard method for the detection of brain microhemorrhage is computed tomography (CT), but magnetic resonance (MR) imaging may be as reliable as CT for microhemorrhage detection in a traumatic brain. ${ }^{2,8)}$ Both CT and MR imaging have been used for the initial radiological evaluation of diffuse axonal injury (DAI). Susceptibility-weighted MR imaging has recently been introduced for the visualization of the venous vasculature and cerebral microbleeds based on the susceptibility difference between deoxyhemoglobin and the surrounding brain tissue. ${ }^{3-5,7,8)}$ Susceptibility-weighted MR imaging uses a high-resolution, completely velocitycompensated, three-dimensional gradient-recalledecho (3D-GRE) sequence based on both magnitude and phase data. Signal change depends on the paramagnetic properties of deoxyhemoglobin in acute hemorrhage and the intravascular spontaneous blood oxygen-level dependent effect. ${ }^{6}$ At our institution, we use the susceptibility-weighted sequence to evaluate patients with DAI or concussion. This technique has shown greater accuracy in hemorrhage detection than conventional MR imag-

Received December 11, 2007; Accepted October 22, 2008 ing techniques, including two-dimensional (2D)GRE $\mathrm{T}_{2}{ }^{*}$-weighted imaging.

The present study retrospectively reviewed 15 patients who underwent CT and MR imaging, including susceptibility-weighted imaging, for initial evaluation of head injury, and compared the sensitivity for hemorrhage detection with CT or conventional MR imaging.

\section{Methods}

Fifteen consecutive patients, 12 males and 3 females aged from 1 to 88 years (mean 36.3 years), with head injury were admitted to our hospital. Consciousness levels ranged from 3 to 14 on the Glasgow Coma Scale. CT and MR imaging were performed within 2 weeks of admission using a LightSpeed Ultra CT scanner (General Electric Co., Fairfield, Conn., U.S.A.) and a 1.5-T Signa ${ }^{\circledR}$ Horizon LX or Signa ${ }^{\circledR}$ Excite EchoSpeed MRI scanner (General Electric Co.). All patients were evaluated using the 3D-GRE susceptibility-weighted sequence in addition to other conventional sequences, including diffusionweighted imaging with apparent diffusion coefficient maps, conventional $\mathrm{T}_{2}$-weighted imaging, and fluid-attenuated inversion-recovery imaging. The susceptibility-weighted imaging parameters were: repetition time, $60 \mathrm{msec}$; echo time, 20-50 
Table 1 Summary of the detection of microhemorrhage in patients with traumatic brain injury

\begin{tabular}{|c|c|c|c|c|c|c|c|c|}
\hline $\begin{array}{l}\text { Case } \\
\text { No. }\end{array}$ & $\begin{array}{l}\text { Age } \\
\text { (yrs) }\end{array}$ & Sex & $\begin{array}{l}\text { Cause of } \\
\text { injury }\end{array}$ & $\begin{array}{l}\text { GCS on } \\
\text { admission }\end{array}$ & $\begin{array}{l}\text { Outcome } \\
\text { (GOS) }\end{array}$ & $\begin{array}{c}\text { No. of lesions } \\
\text { by } \mathrm{T}_{2}{ }^{*}- \\
\text { weighted } \\
\text { imaging }\end{array}$ & $\begin{array}{c}\text { No. of lesions } \\
\text { by } \\
\text { susceptibility- } \\
\text { weighted } \\
\text { imaging }\end{array}$ & $\begin{array}{c}\text { Time of MR } \\
\text { imaging after } \\
\text { injury } \\
\text { (day) }\end{array}$ \\
\hline 1 & 4 & M & pedestrian & 5(E1V1M3) & GR & 24 & 77 & 6 \\
\hline 2 & 5 & M & pedestrian & 6(E1V1M4) & SD & 17 & 154 & 12 \\
\hline 3 & 22 & M & fall & 4(E1V1M2) & SD & 40 & 117 & 21 \\
\hline 5 & 40 & M & TA, driver & 7(E1V2M4) & GR & 28 & 66 & 7 \\
\hline 6 & 44 & M & TA, driver & 8(E1V3M4) & MD & 8 & 88 & 12 \\
\hline 7 & 55 & M & TA, driver & 8(E1V3M4) & $\mathrm{MD}$ & 5 & 52 & 10 \\
\hline 8 & 57 & $\mathrm{~F}$ & TA, driver & 6(E1V1M4) & $\mathrm{SD}$ & 18 & 105 & 12 \\
\hline 9 & 5 & M & TA, passenger & 6(E1V1M4) & GR & 15 & 20 & 7 \\
\hline 10 & 88 & M & TA, passenger & 6(E1V1M4) & VS & 60 & 178 & 8 \\
\hline 15 & 21 & M & TA, driver & 3(E1V1M1) & VS & 55 & 120 & 10 \\
\hline
\end{tabular}

GCS: Glasgow Coma Scale, GOS: Glasgow Outcome Scale, GR: good recovery, MD: moderately disabled, MR: magnetic resonance, SD: severely disabled, TA: traffic accident, VS: vegetative state.

(mean 40) msec; flip angle, $20^{\circ}$; receiver bandwidth, $11.9-25 \mathrm{kHz}$; matrix, $320 \times 192$; number of slices, 32-44; and scan time, 0658-0812. SWIRecon software (especially developed by the manufacturer) was used for reconstruction and the minimum threshold was 0.08 . In addition, a multi 9 filter matrix $(64 \times 64)$ was used. The numbers of hypointense spotty lesions were counted on $\mathrm{T}_{2}{ }^{*}$-weighted and susceptibility-weighted images.

\section{Results}

Hemorrhage was detected in all 15 patients with brain injury (Table 1). Susceptibility-weighted imaging demonstrated hypointense spotty lesions in the injured brain, especially in the corpus callosum, in greater numbers than detected by $\mathrm{T}_{2}{ }^{*}$-weighted or conventional MR imaging (Fig. 1). Susceptibilityweighted imaging detected a mean of $76 \pm 52$ (total 1132) hypointense spotty lesions, compared to a mean of $21 \pm 19$ (total 316) detected by $\mathrm{T}_{2}{ }^{*}$-weighted imaging ( $\mathrm{p}<0.0001$, paired t-test).

\section{Discussion}

2D-GRE $\mathrm{T}_{2}{ }^{*}$-weighted MR imaging can identify intracranial hemorrhage by detecting the presence of deoxyhemoglobin, which was believed to take up to 24 hours to develop post-hemorrhage. However, the transition to deoxyhemoglobin occurs within the first few hours of the development of cerebral hema-
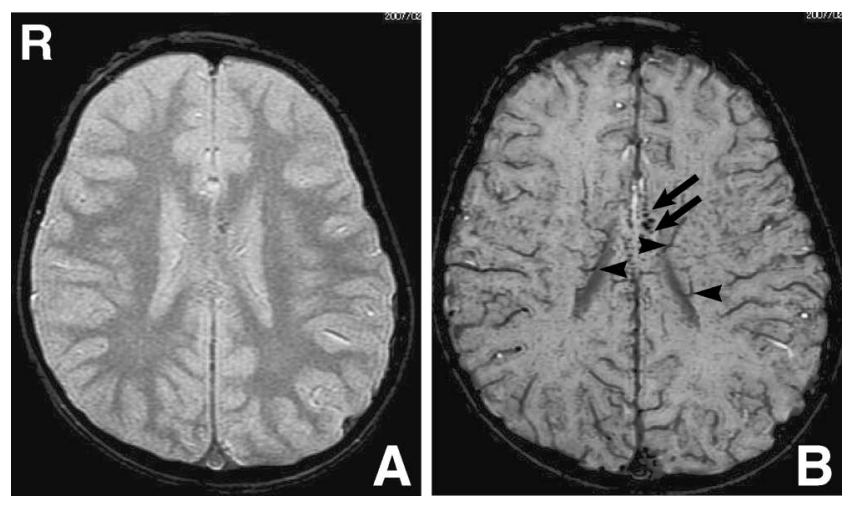

Fig. $1 \mathrm{~T}_{2}{ }^{*}$-weighted magnetic resonance (MR) image (A) showing some spotty microhemorrhages. Susceptibility-weighted MR image (B) showing many more cerebral microhemorrhages as hypointense spotty lesions in the corpus callosum (arrows), which were distinct from the ventricular vessels (arrowheads).

toma, and can be detected as hypointensity earlier on $\mathrm{T}_{2}{ }^{*}$-weighted MR imaging compared to other MR imaging sequences. ${ }^{1,7)}$ In this study, we demonstrated that susceptibility-weighted MR imaging was more sensitive than $\mathrm{T}_{2}{ }^{*}$-weighted $\mathrm{MR}$ imaging. However, slow venous blood flow may appear similar to spotty hemorrhage, so hemorrhage should be carefully distinguished from venous spots.

Susceptibility-weighted imaging utilizes the magnetic susceptibility effects that result from local in- 
homogeneities of the magnetic field. Therefore, pathologies located close to air-filled cavities, such as the sinus or brain surface, cannot be detected because the signal differences between the brain and the external surface are not evident due to artifacts derived from air signals. The present results suggest that more sensitive imaging techniques such as susceptibility-weighted MR imaging might be better predictor of outcomes by providing early evidence of injury or microhemorrhage. However, the sample size in this study was extremely small, so further studies are required.

\section{References}

1) Atlas SW, Thulborn KR: MR detection of hyperacute parenchymal hemorrhage of the brain. AJNR Am J Neuroradiol 19: 1471-1507, 1998

2) Bradley WG Jr: MR appearance of hemorrhage in the brain. Radiology 189: 15-26, 1993

3) Fiebach JB, Schellinger PD, Gass A, Kucinski T, Siebler M, Villringer A, Olkers P, Hirsch JG, Heiland S, Wilde P, Jansen O, Röther J, Hacke W, Sartor K; Kompetenznetzwerk Schlaganfall B5: Stroke magnetic resonance imaging is accurate in hyperacute intracerebral hemorrhage: a multicenter study on the validity of stroke imaging. Stroke 35: 502-506, 2004

4) Liang L, Korogi Y, Sugahara T, Shigematsu Y, Okuda T, Ikushima I, Takahashi M: Detection of intracranial hemorrhage with susceptibility-weighted MR sequences. AJNR Am J Neuroradiol 20: 1527-1534, 1999

5) Linfante I, Llinas RH, Caplan LR, Warach S: MRI features of intracerebral hemorrhage within 2 hours from symptom onset. Stroke 30: 2263-2267, 1999

6) Ogawa S, Lee TM, Kay AR, Tank DW: Brain magnetic resonance imaging with contrast dependent on blood oxygenation. Proc Natl Acad Sci U S A 87: 9868-9872, 1990

7) Patel MR, Edelman RR, Warach S: Detection of hyperacute primary intraparenchymal hemorrhage by magnetic resonance imaging. Stroke 27: 2321-2334, 1996

8) Schellinger PD, Jansen O, Fiebach JB, Hacke W, Sartor K: A standardized MRI stroke protocol: comparison with CT in hyperacute intracerebral hemorrhage. Stroke 30: 765-768, 1999

Address reprint requests to: Yukinori Akiyama, M.D., Department of Neurological Surgery, Sapporo Medical University, South 1 West 16, Chuo-ku, Sapporo, Hokkaido 060-8543, Japan.

e-mail: akiyuki@sapmed.ac.jp

\section{Commentary}

In regard to this issue, it is very essential to define the term "microbleeding" clearly. To evaluate "microbleeding," microscopic examination is essential. In axonal injury, not only axons but also vessels surrounding axons are collapsed by traumatic forces. Necessarily, vascular damages always coexist with axonal injury. Therefore, I think size of hemorrhage must be evaluated not only by images but also with microscopical findings.

In the case shown in Figure 1, which is supposed to be in a vegetative state, volume of bleeding seems to be rather exaggerated, possibly because of artifacts on images. But it is possible that susceptibility-weighted imaging (SWI) emphasizes venous systems excessively. According to Table 1, clinical outcome is shown only with scores of Glasgow Outcome Scale. Although SWI detects more lesions than other MR methods, differences in diagnosis between SWI and other imaging methods are not clear. It can be indicated SWI is not necessarily crucial to evaluate outcome. You must stress the advantage and necessity of SWI. The outcome must be examined not only with Glasgow Outcome Scale, but from multidimensional points of view.

SWI is suggested to have exceptional sensitivity in diagnostic imaging. At present, numbers of cases, including distribution of ages are not adequate. However, this study will grow to be more fruitful through further investigation with concrete evidence.

Takeki OGAWA, M.D.

Department of Emergency Medicine The Jikei University School of Medicine

Tokyo, Japan

Susceptibility-weighted magnetic resonance (MR) imaging has recently been introduced for the visualization of the venous vasculature and cerebral microhemorrhages. In this paper, the authors compared the sensitivity of susceptibility-weighted MR imaging with conventional MR imaging and CT for detection of cerebral hemorrhages in patients with head injury. The authors found that susceptibility-weighted imaging is extremely sensitive for the visualization and detection of microhemorrhages. To find tissue tear hemorrhages is very important for the diagnosis of diffuse axonal injury (DAI), in particular hemorrhages in the corpus callosum or dorsal part of brainstem. The CT and MR imaging demonstration of DAI detects small focal tissue tear hemorrhages in the white matter, basal ganglia, corpus callosum, or dorsal part of brainstem, and the size of these tissue tear hemorrhages is mainly between 0.5 and $1.5 \mathrm{~cm}$. As we know, CT scans or convention MR imaging fail to demonstrate small hemorrhages in some DAI patients. The susceptibility-weighted imaging technique may show greater accuracy for small hemorrhages and improve the diagnostic level of DAI.

In this paper, the number of patients is small, so further studies are required to collect more cases. The MR imaging investigation extended from 4 to 21 days after injury, but no patients within 4 days. We want to know whether it is sensitive within 4 days as for from 4 to 21 days after injury for the detection of microhemorrhages.

Shuyuan YANG, M.D. Department of Neurosurgery Tianjin Medical University General Hospital Tianjin, P.R.C. 